\title{
Integrated Racetrack Micro-Resonator Based on Porous Silicon Ridge Waveguides
}

\author{
L. Poffo ${ }^{1^{*}}$, P. Girault ${ }^{1}$, N. Lorrain ${ }^{1}$, J. Lemaitre ${ }^{1}$, M. Guendouz ${ }^{1}$, \\ P. Azuelos ${ }^{1}$, I. Hardy ${ }^{1,2}$, A. Gutierrez ${ }^{1}$, L. Bodiou ${ }^{1}$, M. Thual ${ }^{1}$, and J. Charrier ${ }^{1}$ \\ ${ }^{1}$ UMR FOTON, CNRS, Université de Rennes 1, Enssat, F22305, Lannion, France \\ ${ }^{2}$ Institut Mines Telecom, Telecom Bretagne, F29238, Brest, France \\ Corresponding authors: *luiz.poffo@univ-rennes1.fr
}

\begin{abstract}
Racetrack micro-resonator (MR), made from partially or totally oxidized porous silicon (PS) ridge waveguides fabricated with standard photolithography process, is reported. The design and the technological process are described including a study of waveguide dimensions that provide single mode propagation. Scanning electronic microscopy observations and optical characterizations clearly show that the MR based on PS ridge waveguides has been well implemented. As the MRs will be used for sensing application, we also present a preliminary theoretical study of the porous MRs sensitivity. A very promising theoretical sensitivity around $1200 \mathrm{~nm} / \mathrm{RIU}$ (Refractive Index Unit) has been calculated for such porous racetrack MR.
\end{abstract}

\section{INTRODUCTION}

Materials based on PS have been widely studied for chemical and biological sensors [1]. Indeed, these materials have significant advantages for use in the fields of sensors thanks to their wide internal surface, as well as their biocompatibility. For example, partially or totally oxidized PS components which have been used for sensing applications are micro-cavities, Bragg reflectors, planar ARROWs, integrated Mach-Zehnder interferometers, and recently, integrated MRs [2-3]. These last components, by their geometry and operating principle, allow, when used as transducers, to produce very sensitive and miniaturized sensors [4]. The interest of a MR based on PS ridge waveguides is to maximize the interaction between the light of the guided mode and the molecules to be detected and present in the PS "by volume". The porous ridge waveguide constitutes the detection medium which will enhance the sensor sensitivity compared to usual SOI (Silicon On Insulator) or Polymer MRs which rely on the evanescent waves to detect the presence of molecules [5]. Recently, the first PS MR [2] has been fabricated using standard electron beam lithography and reactive ion etching. A detection sensitivity of 380 $\mathrm{nm} / \mathrm{RIU}$ have been obtained when salt water solutions infiltrated the porous device, compared to sensitivities of about $100 \mathrm{~nm} / \mathrm{RIU}$ for conventional MR with the same geometries [4].

In this study, we present the manufacturing of an integrated racetrack MR, made from PS ridge waveguides by using an electrochemical etching method of silicon substrate, followed by partial or total thermal oxidation and then by a standard photolithography process. We first present the fabrication method and the characteristics of the PS layers partially or totally oxidized as well as the photolithography process implemented to elaborate the porous racetrack MR based on ridge waveguides. Then, we briefly report the calculation methods that we have implemented to get the waveguide and ring dimensions required for single mode propagation. Finally, the obtained MRs are structurally and optically characterized.

\section{EXPERIMENTAL RESULTS AND DISCUSSIONS}

The MR structure (Fig. 1a, 1b) based on PS ridge waveguides is made by the formation of two layers of PS with different porosities followed by a thermal oxidation and then a standard photolithography process patterning with polymer photoresist and etching steps (wet and dry). The thermal oxidation is necessary to stabilize the PS structure and can be adapted to obtain a partial or total oxidation of PS. The MR PS structure which is studied in this abstract has been totally oxidized in order to get transparent porous silica ridge waveguides.
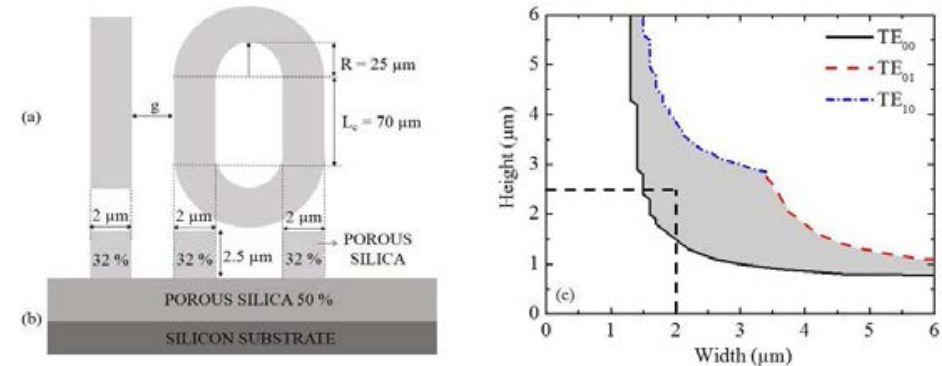

Figure 1. Schema of a top view (a) and a cross section (b) of the porous silica racetrack MR. (c) Solutions of the Maxwell equations for the $T E_{00}, T E_{01}$ and $T E_{10}$ modes as a function of the porous silica ridge waveguide dimensions. 
The dimensions of the porous silica core waveguide $\left(2 \times 2.5 \mu \mathrm{m}^{2}\right)$ shown in Fig. $1 \mathrm{~b}$, have been chosen to provide single mode propagation at $1.55 \mu \mathrm{m}$. The effective refractive index method, involving solutions to the Maxwell equations, and Matlab software were used to calculate the effective refractive index of the ridge porous silica waveguide as a function of its width and height for three different TE modes: the 00 fundamental mode, the 01 mode and the 10 mode. The grey area in Fig. 1c, between the lowest limits of the TE00 mode (fundamental mode) and the TE10 and TE01 modes, represents the single mode region. In Figs. 2a, 2b, 2c and 2d, Scanning Electronic Microscopy (SEM) images of the ridge racetrack porous silica MR are presented. At this magnification, the racetrack has been well achieved. Otherwise, the gap between the straight waveguide and the racetrack waveguide is $0.8 \pm 0.1 \mu \mathrm{m}$ (Fig. $2 \mathrm{~d}$ ), instead of the $0.6 \mu \mathrm{m}$ expected. The real separation distance between the two waveguides is higher than expected by $0.2 \mu \mathrm{m}$ because of the additional lateral etch that occurred at the top of the porous layers.
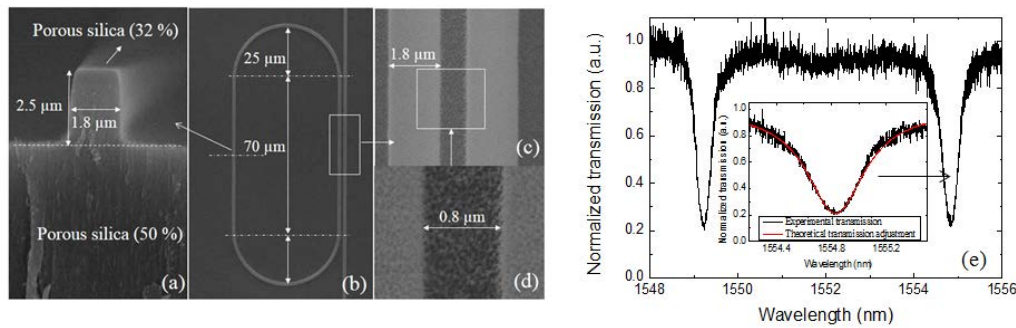

Figure 2. SEM images of the racetrack resonator: (a) cross section of a porous silica ridge waveguide; top views at different scales of: $(b)$ the whole racetrack $M R,(c)$ the two waveguides in the coupling area and $(d)$ the gap separation between the two waveguides of the coupling area. (e) Experimental transmission spectra for one polarization of light. In the insert, the theoretical transmission is adjusted to the experimental one.

In order to verify that the porous silica racetrack MR is optically operational, the spectral transmission responses, for different gaps, were measured. The output from a tunable wavelength laser is injected in the input straight waveguide using a lensed fiber with a mode radius of $2.2 \mu \mathrm{m}$ and constant polarization. A second lensed single mode fiber is also used to couple the output of the straight waveguide to an optical spectrum analyzer. The transmission measurements were done with a step resolution of $1 \mathrm{pm}$ over the wavelength range of $1540-1560 \mathrm{~nm}$ at the output of the straight waveguide. The measured transmission as a function of the wavelength for one polarization of light is reported in Fig. 2e for the target gap of $0.6 \mu \mathrm{m}$. The spectrum shows two resonance wavelengths at $1549 \mathrm{~nm}$ and $1555 \mathrm{~nm}$ in the studied range. This demonstrates that the light is propagated in the waveguide and the coupling of the light occurs between the access waveguide and the MR. This experiment shows clearly that we have succeed in making a ridge porous silica racetrack MR. Finally, in order to estimate the sensitivity of such MR, we have calculated the MRs transmission transfer function considering the grafting of Bovin Serum Albumin (BSA) molecules for different concentration [3]. The theoretical and expected sensitivity of the porous silica MR reaches a value around $1200 \mathrm{~nm} / \mathrm{RIU}$ which corresponds to a sensitivity of $0.02 \mathrm{~nm} / \mathrm{pg} / \mathrm{mm}^{2}$. Such a sensitivity value is high for such single porous silica MR and is very promising for the sensing applications.

\section{CONCLUSION}

Knowing the refractive index of each porous layer, the dimensions of the ridge waveguide to provide single mode propagation were determined. SEM observations and optical characterizations of the porous silica MR showed that the MR had been successfully implemented. Our current work, in progress, is to further optically characterize the resonator and the waveguides in order to determine the optical losses. Then, the porous racetrack MR will be used as a sensor and we will experimentally determine its sensitivity. A promising theoretical sensitivity (about $1200 \mathrm{~nm} / \mathrm{RIU}$ ) of the porous racetrack MR has been obtained [5].

\section{REFERENCES}

[1] J. Gao, T. Gao and M. J. Sailor: Porous-silicon vapor sensor based on laser interferometry, Applied Physics Letters, 2000, 77:901-903.

[2] G.A. Rodriguez, H. Shuren, and S.M. Weiss: Porous silicon ring resonator for compact, high sensitivity biosensing applications, Optics Express, 2015, 23, 6:7111-7119.

[3] P. Girault et al.: Racetrack micro-resonators based on ridge waveguides made of porous silica, Optical Materials, 1-8, 2015.

[4] C. Ciminelli, F. Dell'Olio, D. Conteduca, C.M. Campanella, and M.N. Armenise: High performance SOI microring resonator for biochemical sensing, Optics \& Laser Technology, 59, 60-67, 2014.

[5] P. Girault et al.: Integrated polymer micro-ring resonators for optical sensing applications, Journal of Applied Physics, 2015, 117:104504. 